\title{
Aperiodic tilings, old and new - an overflight
}

Shelomo Ben-Abraham Ben-Gurion University of the Negev

I briefly sketch the background and motivation for studying ordered aperiodic structures. I show a few examples of fivefold tilings relevant to metallic quasicrystals as well as some twelvefold tilings occurring mainly in soft matter. I present my own eightfold spacefilling structure interpreting the structure of the MnSiAl quasiperiodic phase in terms of covering by clusters. By comparison of the Golay-Rudin-Shapiro and Champernowne sequences I demonstrate that nonzero entropy does not imply stochasticity. Eventually I show our own multidimensional generalizations of the Paperfolding and Period Doubling sequences as well as the Chair Code, Brick and Crossed Cube tilings. 